\title{
Toxicidad de los aceites esenciales de Bursera graveolens, Lepechinia meyenii y Myrtus communis sobre Chrysoperla asoralis, Chrysoperla externa y Ceraeochrysa cincta (Neuroptera: Chrysopidae)
}

Toxicity of the essential oils of Bursera graveolens, Lepechinia meyenii
and Myrtus communis against Chrysoperla asoralis, Chrysoperla externa
and Ceraeochrysa cincta

Recibido: noviembre 20 de 2019 | Revisado: diciembre 15 de 2019 | Aceptado: enero 14 de 2020

\author{
Hector Ponce ${ }^{\mathrm{I}}$ \\ José IANNACONE ${ }^{\mathrm{I}, 2}$ \\ Lorena Alvariño ${ }^{1}$ \\ Mario Carhuapoma ${ }^{3}$
}

1 Universidad Nacional Federico Villarreal (UNFV), Escuela Universitaria de Postgrado. Grupo de Investigación Sostenibilidad Ambiental (GISA). Facultad de Ciencias Naturales y Matemática (FCCNM), Laboratorio de Ecología y Biodiversidad Animal (LEBA), Lima, Perú.

2 Universidad Ricardo Palma. Facultad de Ciencias Biológicas. Lima, Perú.

3 Universidad Nacional Mayor de San Marcos (UNMSM), Facultad de Farmacia y Bioquímica (FFB), Lima, Perú.

* Autor para correspondencia E-mail: joseiannacone@gmail.com

\section{Resumen}

El uso excesivo de agroquímicos puede ocasionar contaminación ambiental y desarrollo de plagas resistentes. Por este motivo, se utilizan a las plantas biocidas como una alternativa para el control integrado de plagas. Aquí evaluamos la toxicidad de los aceites esenciales de Bursera graveolens (Burseraceae), Lepechinia meyenii (Lamiaceae) y Myrtus communis (Myrtaceae) sobre los huevos y larvas del primer instar de Chrysoperla externa, Chrysoperla asoralis y Ceraeochrysa cincta (Neuroptera: Chrysopidae) bajo condiciones de laboratorio. Se emplearon diluciones con alcohol etílico al $70 \%$ de B. graveolens y L. meyenii al $20 \%, 10 \%$ y $5 \%$; y con Dimetilsulfóxido (DMSO) de M. communis al $2 \%, 1 \%, 0,5 \%$, $0,25 \%$ y $0,125 \%$, en un período de -120 h para la eclosión de huevos, y de $24 \mathrm{~h}$ y $48 \mathrm{~h}$ de exposición para evaluar toxicidad por contacto residual. Se calcularon los parámetros de toxicidad de LOEC (Concentración más baja con efecto observado), NOEC (Concentración sin efecto observado) y CL50 (Concentración Letal media). Los aceites esenciales de B. graveolens, L. meyenii y M. communis presentaron efecto sobre la eclosión de los huevos de los tres crisópidos. B. graveolens y L. meyenii presentan poco o nulo efecto sobre las larvas de los neurópteros estudiados; mientras que el aceite esencial de $M$. communis, sí presentó efecto larvicida. Los aceites esenciales pueden ser usados en el manejo integrado de plagas.

Palabras clave: aceite esencial, bioensayos, Chrysopidae, plaguicidas botánicos, toxicidad

\section{Abstract}

Excessive use of agrochemicals can cause environmental pollution and development of resistant pests. For this reason, biocidal plants are used as an alternative for integrated pest control. The toxicity of the essential oils of Bursera graveolens (Burseraceae), Lepechinia meyenii (Lamiaceae) and Myrtus communis (Myrtaceae) on the

(C) Los autores. Este artículo es publicado por la Revista Campus de la Facultad de Ingeniería y Arquitectura de la Universidad de San Martín de Porres. Este artículo se distribuye en los términos de la Licencia Creative Commons Atribución No-comercial - Compartir-Igual 4.0 Internacional (https://creativecommons.org/licenses/ CC-BY), que permite el uso no comercial, distribución y reproducción en cualquier medio siempre que la obra original sea debidamente citada. Para uso comercial contactar a: revistacampus@usmp.pe. 
eggs and larvae of the first instar of Chrysoperla externa, Chrysoperla asoralis and Ceraeochrysa cincta (Neuroptera: Chrysopidae) was evaluated under conditions from laboratory. Dilutions with $70 \%$ ethyl alcohol of B. graveolens and $20 \%$ L. meyenii, $10 \%$ and $5 \%$ were used; and with Dimethyl sulfoxide (DMSO) of $M$. communis at $2 \%, 1 \%$, $0.5 \%, 0.25 \%$ and $0.125 \%$, in a period of $-120 \mathrm{~h}$ for egg hatching, and $24 \mathrm{~h}$ and $48 \mathrm{~h}$ of exposure to assess residual contact toxicity. The toxicity parameters of LOEC (lowest concentration with observed effect), NOEC (concentration without observed effect) and LC50 (mean lethal concentration) were calculated. The essential oils of $B$. graveolens, $L$. meyenii and $M$. communis had an effect on the hatching of the eggs of the three chrysopids. B. graveolens and L. meyenii have little or no effect on the larvae of the studied neuroptera; while the essential oil of $M$. communis did show larvicidal effect. Essential oils can be used in integrated pest management.

Key words: botanical pesticides, bioassays, Chrysopidae, essential oil, toxicity

\section{Introducción}

La aplicación de agroquímicos es una de las herramientas más empleadas actualmente para el control de plagas agrícolas, pero, su uso excesivo puede producir contaminación ambiental y desarrollo de plagas resistentes (Sarwar, 2015; Campos et al., 2016) por lo que existe una necesidad urgente de desarrollar alternativas seguras que tengan el potencial de reemplazar los fumigantes tóxicos, pero que sean efectivas, económicas y convenientes de usar (Ayvaz et al., 2008). Por estas razones, los productos derivados de las plantas, como los aceites esenciales, son una fuente de nuevas alternativas con potencial para el control de plagas agrícolas (Souza et al., 2008), debido a su menor toxicidad en el ambiente con relación a los insecticidas sintéticos, efectividad como plaguicida (Cruz et al., 2014) y, además, provienen de fuentes renovables (Tarqui, 2007).

Bursera graveolens (Kunth) Triana \& Palnch, 1872 (Burseraceae), "palo santo" es una planta nativa que crece en bosques secos de la costa pacífica de Sudamérica en gran parte de América. Ha sido utilizada desde épocas remotas como remedio para dolores estomacales, sudoríficos y como linimento para reumatismos y fuertes fiebres. El aceite esencial de la corteza de $B$. graveolens presentan actividad fungicida contra hongos fitopatógenos como Botrytis cinerea y Fusarium oxysporum. También es usado para tratar (tópicamente) la fatiga, jaqueca, mareos y taquicardia (UICN, 2019).

Lepechinia meyenii Epling, 1935 (Lamiaceae), "pacha salvia" es una planta nativa de los Andes occidentales, distribuida en Argentina, Bolivia y Perú, entre los 1500 y 4500 msnm. Como infusión se utiliza para combatir bronquitis, problemas del corazón, de nervios. Combinado con Rosmarinus officinalis y Plantago linearis, se utiliza para curar heridas y pérdida del cabello (Bussmann \& Sharon, 2015). En el Callejón de Huaylas y en la Región de los Libertadores Wari, los pobladores la usan como infusión de planta entera sola o combinada con culén y orégano blanco para el tratamiento de diarreas, cólicos, afecciones hepáticas y como 
aperitivo (Rossi et al., 2002). Además, presenta sustancias antioxidantes (Castillo, 2004).

Myrtus communis Linneo, 1753 (Myrtaceae), "arrayán" o "mirto" es una planta nativa del sur de Europa, también se encuentra en Ecuador, Colombia, Costa Rica, Panamá, Perú y Venezuela (Guzmán, 2005). Sus propiedades aromáticas se deben a los principios activos, componentes descubiertos en sus hojas (Gardeli et al., 2008), donde aceites esenciales, taninos, triterpenos y algunos tipos de glucósidos como los flavonoides han sido encontrados en cantidades importantes (Satrani et al., 2006), componentes que a su vez, le otorgan propiedades antisépticas y antimicrobianas en especial para bacterias gran positivas, convirtiendo al arrayán como un elemento adecuado en el control de problemas respiratorios, disentería, estomatitis, faringitis, psoriasis, herpes (Berka-Zougali et al., 2012). Se ha registrado la toxicidad del aceite esencial de $M$. communis sobre diferentes insectos plaga como larvas y adultos de Trogoderma granarium (Tayoub et al., 2012), adultos de Tribolium confusum y Callosobuchus aculatus (Khani \& Basavand, 2012), Tetranychus urticae (Motazedian et al., 2012), mostrando efectividad como insecticida.

Chrysoperla externa Hagen, 1861 (Chrysopidae), "león de áfidos" destaca como controlador biológico por su gran voracidad en estado larval, amplia distribución, presencia de adultos todo el ańo, fácil crianza en cautiverio, potencial para adaptarse a varios ambientes de cultivo y su resistencia a numerosos plaguicidas (Silva et al., 2012; Moura et al., 2012), así como a productos botánicos
(Iannacone \& Lamas, 2002; Medina et al., 2004; Iannacone et al., 2015; Alegre et al., 2017ab) y se le ha registrado depredando un amplio rango de insectos de cuerpo blando, especialmente huevos y larvas de lepidópteros. Está considerada en programas de Manejo Integrado de Plagas (MIP) del Programa Nacional de Control Biológico (PNCB), se producen masivamente en los laboratorios de control biológico del Perú, para su distribución y sostenimiento de programas de MIP (Nuñez, 1988).

Chrysoperla asoralis Banks, 1915 (Chrysopidae), es una especie depredadora perteneciente de la región Neotropical en donde ha sido utilizada con mucho éxito para el control de plagas en hortalizas, como los espárragos (Gonzáles \& Reguillón, 2002) y frutales tanto en campo abierto como en invernadero. Las condiciones climáticas del Perú, especialmente la costa (donde hay ausencia de lluvias y temperaturas favorables para las crisopas durante todo el año), provocan el gran éxito de este depredador.

Ceraeochrysa cincta Schneider, 1851 (Chrysopidae), está ampliamente distribuida desde Florida, Estados Unidos de América (EUA), hasta Argentina, incluyendo las Islas Galápagos en América del Sur (Ramírez et al., 2007). Por la gran distribución y asociación con diversos artrópodos plaga en diferentes sistemas agrícolas, se considera que esta especie presenta un gran potencial como agente de control biológico (Iannacone \& Alvariño, 2010).

El conocer los efectos de los insecticidas botánicos sobre los controladores biológicos ayudarían a comprobar su 
compatibilidad para su posible uso en el MIP (Iannacone et al., 2015; Parolín et al., 2015; Alegre et al., 2017a). El objetivo de este trabajo es evaluar la toxicidad de los aceites esenciales de $B$. graveolens, $L$. meyenii y $M$. communis sobre $C$. asoralis, C. externa y $C$. cincta, bajo condiciones de laboratorio.

\section{Método}

Los bioensayos se realizaron en el Laboratorio de Ecología y Biodiversidad Animal (LEBA) de la Facultad de Ciencias Naturales y Matemática de la Universidad Nacional Federico Villarreal (UNFV), El Agustino, Lima, Perú, durante el 2019.

\section{Aceites esenciales}

Los aceites esenciales de $B$. graveolens y L. meyenii al 70\%, y de $M$. communis al $100 \%$ se extrajeron por arrastre de vapor de agua. Estos aceites fueron adquiridos del Centro de Investigación de Plantas Medicinales Aromáticas y Medicina Tradicional (CIPLAMT). Se realizaron diluciones con alcohol al $70 \%$ de los aceites de $B$. graveolens y L. meyenii al 20\%, $10 \%$ y $5 \%$; y con Dimetilsulfóxido (DMSO) del aceite de M. communis al $2 \%, 1 \%, 0.5 \%, 0.25 \%$ y $0.125 \%$ (Barrueta et al., 2017; Sariol et al., 2019). Finalmente, las diluciones se almacenaron en frascos de color ámbar para su uso en los bioensayos.

\section{Insectos}

Se adquirieron huevecillos maduros de $C$. externa, $C$. asoralis y de $C$. cincta del Programa Nacional de Control Biológico, Servicio Nacional de Sanidad Agraria (PN-CB- SENASA), Lima, Perú, los cuales se separaron para ensayos de inmersión y para los ensayos de contacto residual. Estos últimos se acondicionaron en recipientes de plástico hasta el momento de su eclosión. Las larvas del primer instar recién emergidas fueron alimentadas con huevos de Sitotroga cerealella (Olivier, 1789) antes de utilizarlos en los bioensayos (Iannacone \& Alvariño, 2010).

\section{Bioensayos}

Los bioensayos por inmersión se llevaron a cabo sumergiendo los huevecillos de los neurópteros durante cinco segundos en las diluciones de los aceites. Posteriormente, los huevos fueron colocados en papel tissue por 10 min para absorber los restos de soluciones acuosas y permitir el secado a temperatura ambiente (Alegre et al., 2017b). Se trataron 10 huevos por cada concentración. Los huevos fueron individualizados en envases de plástico de $12 \mathrm{~mL}$ de capacidad. Después de las aplicaciones tópicas, los envases de plástico se mantuvieron en oscuridad bajo condiciones de cría, realizándose las lecturas hasta la eclosión de los huevos $(\sim 120 \mathrm{~h})$.

Los bioensayos por contacto residual se llevaron a cabo en envases de plástico de $12 \mathrm{~mL}$ de capacidad, a los cuales se les hizo pequeños orificios en la tapa para permitir su respiración (Iannacone et al., 2015; Celaya et al., 2019; Valdiviezo, 2019). Se pasó un hisopo estéril previamente sumergido por 30 seg una sola vez en cada extracto vegetal, para luego esparcirlo homogéneamente por todas las paredes de cada recipiente plástico. Luego, los envases se secaron a temperatura ambiente por un intervalo de 2 h (Iannacone \& Alvarińo, 2010). Diez 
larvas del primer instar de los neurópteros se colocaron individualmente en cada recipiente plástico (Alegre et al., 2017b), debido a que estos insectos presentan hábitos de canibalismo cuando escasea el alimento (Núñez, 1988). Los bioensayos se realizaron en condiciones de oscuridad para evitar la fotólisis de los extractos botánicos (Iannacone \& Lamas, 2003; Iannacone \& Alvariño, 2005). Las lecturas de mortalidad fueron a las $24 \mathrm{~h}$ y $48 \mathrm{~h}$. Se evidenció la muerte cuando la larva no realice algún movimiento corporal coordinado al ser estimulado con un alfiler entomológico durante 15 seg en su observación en el microscopio estereoscópico (Iannacone \& Alvariño, 2005).

\section{Diseño experimental y análisis de datos}

Se usaron tres concentraciones (20\%, $10 \%$ y $5 \%)$ del aceite esencial de $B$. graveolens y $L$. meyenii y cinco concentraciones $(2 \%, 1 \%, 0,5 \%, 0,25 \%$ y $0,125 \%$ ) de $M$. communis, cada uno con cuatro repeticiones, un control de agua destilada para los dos primeros aceites y un control de DMSO para el último. Se realizó el análisis de varianza (ANOVA) y la prueba post hoc de Tukey con un nivel de significancia de $\mathrm{p} \leq 0,05$. Se hizo un ajuste del control a $0 \%$ mediante la fórmula de Schneider-Orelli's derivada de la fórmula de Abbot (Fusé et al., 2013). Se calcularon los valores de LOEC (Concentración más baja con efecto observado) y NOEC (Concentración sin efecto observado) según Alegre et al. (2017b) para los efectos de toxicidad de los tres aceites. Se calculó la
CL50 usando el programa computarizado Probit versión 1.5 (Weber, 1993) para los efectos del aceite de $M$. communis. Se empleó el paquete estadístico SPSS, versión 25 para Windows 10 (2018) para el cálculo de los estadísticos descriptivos e inferenciales.

\section{Resultados y discusión}

Los aceites esenciales de $B$. graveolens y $L$. meyenii presentaron efecto sobre la eclosión de los huevos de C. externa, $C$. asoralis y $C$. cincta en las tres concentraciones. Los valores de LOEC y NOEC fueron de $5 \mathrm{~g} \cdot 100 \mathrm{~mL}^{-1}$ y $<5 \mathrm{~g} \cdot 100 \mathrm{~mL}^{-1}$, respectivamente para los efectos de los extractos sobre los tres neurópteros (Tabla 1). El aceite esencial de $M$. communis presentó efecto sobre la eclosión de los huevos de los tres controladores biológicos siendo $C$. asoralis la especie más sensible, seguido de C. cincta y C. externa (Tabla 2).

El efecto ovicida de estos aceites puede ocurrir debido a una toxicidad aguda o a la alteración de procesos fisiológicos y químicos del desarrollo embrionario por la penetración de los compuestos volátiles de los aceites dentro de los huevos (Ramírez et al., 2010), aunque los autores mencionan que los huevos de algunas especies presentan mayor tolerancia a agentes químicos en comparación a otros estados de desarrollo del insecto (González et al., 2016). Sin embargo, para asegurar lo que ocasiona el efecto ovicida por parte de los aceites utilizados, es recomendable realizar pruebas complementarias. 
Tabla 1

Efecto de Bursera graveolens y Lepechinia meyenii sobre Chrysoperla externa, Chrysoperla asoralis y Ceraeochrysa cincta (Neuroptera: Chrysopidae) en términos de porcentaje de no eclosión de huevos.

\begin{tabular}{ccccccc}
\hline & \multicolumn{2}{c}{ C. externa } & \multicolumn{2}{c}{ C. asoralis } & \multicolumn{2}{c}{ C. cincta } \\
Concentración & B. graveolens & L. meyenii & B. graveolens & L. meyenii & B. graveolens & L. meyenii \\
\hline Control & $0 \mathrm{a}$ & $0 \mathrm{a}$ & $0 \mathrm{a}$ & $0 \mathrm{a}$ & $0 \mathrm{a}$ & $0 \mathrm{a}$ \\
$5 \%$ & $92,1 \mathrm{~b}$ & $92,3 \mathrm{~b}$ & $86,4 \mathrm{~b}$ & $95 \mathrm{~b}$ & $87,2 \mathrm{~b}$ & $97,5 \mathrm{~b}$ \\
$10 \%$ & $97,4 \mathrm{~b}$ & $94,8 \mathrm{~b}$ & $91,8 \mathrm{~b}$ & $100 \mathrm{~b}$ & $97,4 \mathrm{~b}$ & $97,5 \mathrm{~b}$ \\
$20 \%$ & $100 \mathrm{~b}$ & $100 \mathrm{~b}$ & $100 \mathrm{~b}$ & $100 \mathrm{~b}$ & $97,4 \mathrm{~b}$ & $100 \mathrm{~b}$ \\
NOEC $(\%)$ & $<5$ & $<5$ & $<5$ & $<5$ & $<5$ & $<5$ \\
LOEC $(\%)$ & 5 & 5 & 5 & 5 & 5 & 5 \\
F & 225,11 & 420 & 108,69 & 1163 & 113,22 & 774 \\
Sig. & 0,00 & 0,00 & 0,00 & 0,00 & 0,00 & 0,00 \\
\hline
\end{tabular}

Letras minúsculas iguales en una misma columna representan que no hay diferencia significativa entre los valores por la prueba de Duncan $(\mathrm{p} \leq 0,05)$. NOEC $=$ Concentración sin efecto observado. LOEC=Concentración mínima con efecto observado. F=Prueba de Fisher. Sig.=Nivel de significancia.

Tabla 2

Efecto de Myrtus communis sobre Chrysoperla externa, C. asoralis y Ceraeochrysa cincta en términos de porcentaje de no eclosión de huevos

\begin{tabular}{cccc}
\hline & C. externa & C. asoralis & C. cincta \\
\hline Concentración & \multicolumn{4}{c}{ Myrtus communis } \\
Control & 0 & $0 \mathrm{a}$ & $0 \mathrm{a}$ \\
$0,125 \%$ & $0 \mathrm{a}$ & $2,86 \mathrm{ab}$ & $0 \mathrm{a}$ \\
$0,25 \%$ & $7,89 \mathrm{ab}$ & $25,71 \mathrm{abc}$ & $5 \mathrm{ab}$ \\
$0,5 \%$ & $10,53 \mathrm{ab}$ & $37,14 \mathrm{c}$ & $10 \mathrm{ab}$ \\
$1 \%$ & $18,42 \mathrm{ab}$ & $31,43 \mathrm{bc}$ & $15 \mathrm{~b}$ \\
$2 \%$ & $26,32 \mathrm{~b}$ & $34,29 \mathrm{c}$ & $32,5 \mathrm{c}$ \\
NOEC $(\%)$ & 1 & 0,25 & 0,5 \\
LOEC $(\%)$ & 2 & 0,5 & 1 \\
CL50 $(\%$ extracto) & 12,15 & 2,81 & 5,20 \\
F & 3,79 & 6,53 & 22,89 \\
Sig & 0,01 & 0,001 & 0,000 \\
\hline
\end{tabular}

Letras minúsculas iguales en una misma columna representan que no hay diferencia significativa entre los valores por la prueba de Duncan $(p \leq 0,05)$. NOEC $=$ Concentración sin efecto observado. LOEC=Concentración mínima con efecto observado. CL50= Concentración Letal Media. F=Prueba de Fisher. Sig.=Nivel de significancia.

El aceite esencial de B. graveolens no presentó efecto insecticida sobre las larvas del primer estadio de C. externa y
C. asoralis a las $24 \mathrm{~h}$ y $48 \mathrm{~h}$ de exposición, de igual manera, el aceite de $L$. meyenii tampoco presenta efecto insecticida sobre 
C. asoralis, pues los resultados obtenidos no difirieron significativamente del control $(p \leq 0,005)$. Sin embargo, el extracto de $L$. meyenii sí presentó efecto larvicida sobre C. externa a las $48 \mathrm{~h}$ de exposición (Tabla 3).

Tabla 3

Efecto de Bursera graveolens y Lepechinia meyenii sobre Chrysoperla externa y Chrysoperla asoralis (Neuroptera: Chrysopidae) en términos de porcentaje de mortalidad larvaria del primer estadio a 24 hy 48 h de exposición.

\begin{tabular}{ccccccccc}
\hline & \multicolumn{3}{c}{ Chrysoperla externa } & \multicolumn{3}{c}{ Chrysoperla asoralis } \\
& B. graveolens & L. meyenii & B. graveolens & L. meyenii \\
& & & \multicolumn{7}{c}{ Exposición } \\
\hline \hline Concentración & $24 \mathrm{~h}$ & $48 \mathrm{~h}$ & $24 \mathrm{~h}$ & $48 \mathrm{~h}$ & $24 \mathrm{~h}$ & $48 \mathrm{~h}$ & $24 \mathrm{~h}$ & $48 \mathrm{~h}$ \\
Control & $0 \mathrm{a}$ & $0 \mathrm{a}$ & $0 \mathrm{a}$ & $0 \mathrm{a}$ & $0 \mathrm{a}$ & $0 \mathrm{a}$ & $0 \mathrm{a}$ & $0 \mathrm{a}$ \\
$5 \%$ & $0 \mathrm{a}$ & $0 \mathrm{a}$ & $0 \mathrm{a}$ & $0 \mathrm{a}$ & $0 \mathrm{a}$ & $0 \mathrm{a}$ & $0 \mathrm{a}$ & $2,5 \mathrm{a}$ \\
$10 \%$ & $0 \mathrm{a}$ & $2,5 \mathrm{a}$ & $2,5 \mathrm{a}$ & $7,5 \mathrm{ab}$ & $0 \mathrm{a}$ & $0 \mathrm{a}$ & $2,5 \mathrm{a}$ & $7,5 \mathrm{a}$ \\
$20 \%$ & $0 \mathrm{a}$ & $2,5 \mathrm{a}$ & $5 \mathrm{a}$ & $12,5 \mathrm{~b}$ & $0 \mathrm{a}$ & $2,5 \mathrm{a}$ & $0 \mathrm{a}$ & $7,5 \mathrm{a}$ \\
NOEC $(\%)$ & 20 & 20 & 20 & 10 & 20 & 20 & 20 & 20 \\
LOEC $(\%)$ & $>20$ & $>20$ & $>20$ & 20 & $>20$ & $>20$ & $>20$ & $>20$ \\
F & 0 & 0,66 & 1,57 & 5.14 & 0 & 1 & 1 & 1,08 \\
Sig. & 0 & 0,58 & 0,24 & 0,01 & 0 & 0,42 & 0,42 & 0,39 \\
\hline
\end{tabular}

Letras minúsculas iguales en una misma columna representan que no hay diferencia significativa entre los valores por la prueba de Duncan $(\mathrm{p} \leq 0.05)$. NOEC $=$ Concentración sin efecto observado. LOEC=Concentración mínima con efecto observado. F=Prueba de Fisher. Sig.=Nivel de significancia.

El efecto del aceite esencial de $L$. meyenii sobre los crisópidos en este trabajo puede deberse a su composición química que presenta $\alpha$-pineno $(21,37 \%)$ y eucaliptol $(18,80 \%)$ como componentes mayoritarios (Quispe \& Tenorio, 2018). Ruiz et al. (2015) también registraron a $\alpha$-Pineno $(29,87 \%)$ y Eucaliptol $(13,25 \%)$ como componentes mayoritarios. Los pinenos han demostrado tener acción ovicida, larvicida y repelente en mosquitos como Aedes aegypti, Culex quinquefasciatus y Anopheles stephensi (Leyva et al., 2009); así mismo, el eucaliptol (1,8-cineol) es un monoterpeno encontrado también en Lavandula spica (Lamiaceae), que presenta actividad insecticida frente
Ceratitis capitata "Mosca mediterránea de la fruta" (Clemente et al., 2007) o Trialeurodes vaporariorum "Mosca blanca" (Choi et al., 2003; García et al., 2014).

Con respecto a $B$. graveolens, tiene secreciones de resinas de monoterpenos y sesquiterpenos en los tallos como protección frente al ataque de los insectos (Aldana et al., 2010). Presenta como composición química a limoneno $(48,2 \%)$ y mirceno $(19,8 \%)$ como componentes mayoritarios (Leyva et al., 2007). El limoneno es reconocido por diversos autores como una sustancia con efecto antialimentario, repelente e insecticida que inhiben el 
desarrollo y crecimiento de muchos insectos, como también actividades antibacteriana y antimicótica. Muñoz et al. (2014) menciona al limoneno como constituyente de plantas colombianas con efecto insecticida como Lippia alba (Verbenaceae), Citrus sinensis (Rutaceae) y Cymbopogon nardus (Poaceae). El mirceno es un monoterpeno encontrado en Schinus molle (Anacardinaceae) que presenta actividad insecticida (Arias et al., 2017). Martínez et al. (2014) utiliza a $B$. graveolens junto con otros extractos vegetales frente a áfidos de la habichuela
(Aphis craccivora) y logró disminuir más del 60\% de la población de los áfidos.

$\mathrm{Al}$ evaluar el efecto de contacto residual del aceite esencial de $M$. communis sobre las larvas del primer instar de los tres controladores biológicos a las cinco concentraciones entre $0,125 \%$ a $2 \%$ se observó efecto un insecticida, excepto para C. externa a las $24 \mathrm{~h}$, pues el resultado no difirió significativamente del control $(\mathrm{p} \leq 0,005)$. Laslarvas de C. asoralis resultaron ser más sensibles al aceite esencial, pues obtuvo valor de CL50 de 1,29\% a las $48 \mathrm{~h}$ de exposición (Tabla 4).

Tabla 4

Efecto de Myrtus communis sobre Chrysoperla externa, Chrysoperla asoralis y Ceraeochrysa cincta en términos de porcentaje de mortalidad larvaria del primer estadio a $24 \mathrm{~h}$ y $48 \mathrm{~h}$ de exposición.

\begin{tabular}{ccccccc}
\hline & \multicolumn{2}{c}{ C. externa } & \multicolumn{2}{c}{ C. asoralis } \\
Myrtus communis \\
Exposición
\end{tabular}

Letras minúsculas iguales en una misma columna representan que no hay diferencia significativa entre los valores por la prueba de Duncan $(p \leq 0,05)$. NOEC $=$ Concentración sin efecto observado. LOEC=Concentración mínima con efecto observado. CL50= Concentración Letal Media. F=Prueba de Fisher. Sig.=Nivel de significancia.

Myrtus communis presentó efecto tóxico sobre los tres controladores biológicos utilizados en este ensayo, tanto para los huevos como para las larvas del primer instar, esto puede deberse a la composición química del aceite esencial, principalmente a los monoterpenos que se encuentran en el aceite (Zayzafoon et al., 2011; Tayoub et al., 2012). Khani $\&$ Bassavand (2012) señalaron como 
componentes principales a $\alpha$-pineno $(10,4 \%), \alpha$-terpineol $(10,1 \%)$, linalool (9,0\%) y 1,8-cineol (7,6\%). Rasooli et al. (2002) menciona al $\alpha$-pineno $(29,4 \%)$, limoneno $(21,2 \%), \quad 1,8$-cineol (18\%) y linalool $(10,6 \%)$ como componentes mayoritarios. Así mismo, Tuberoso et al. (2006) mencionan que los principales compuestos en el aceite esencial de las hojas de $M$. communis fueron $\alpha$-pineno $(30,0 \%), 1,8$-cineol $(28,8 \%)$ y limoneno $(17,5 \%)$. Los monoterpenoides son típicamente compuestos volátiles y bastante lipofílicos, que pueden penetrar rápidamente en los insectos e interferir con sus funciones fisiológicas (Lee et al., 2002) actuando como repelente o insecticida (Bakkali et al., 2008). Es posible que el aceite esencial de $M$. communis, con sus componentes monoterpenoides, actúe contra los insectos como neurotoxinas (Papachristos \& Stamopoulos, 2004; Ayvaz et al., 2010). Se sugirió que los terpenos naturales aislados de aceites esenciales podrían actuar como activadores de los receptores octopaminérgicos en las larvas de Trogoderma granarium (Kostyukovsky et al., 2002).

Las larvas de los tres controladores biológicos utilizados en este ensayo presentaron respuestas diferentes frente a los tres aceites esenciales empleados, la diferencia de sensibilidad puede atribuirse principalmente a la resistencia fenotípica (modificaciones en el sitio objetivo), la resistencia metabólica (capacidad de desintoxicar insecticidas) o la modificación del comportamiento.
Los cambios de comportamiento que minimizan el contacto entre el insecto y el insecticida pueden causar un impacto severo en la eficacia de la aplicación del insecticida, especialmente si las características fisiológicas seleccionan la resistencia (Martins \& Valle, 2012).

Estudios han demostrado que los aceites esenciales y sus componentes pueden utilizarse potencialmente como compuestos alternativos a los fumigantes sintéticos en uso actual (Shakarami et al., 2005; Ayvaz et al., 2010, Tayoub et al., 2012; Khani \& Bassavand, 2012; Alegre et al., 2017a), por lo que los aceites esenciales manejados en el presente trabajo podrían convertirse en una alternativa interesante a las estrategias convencionales de control químico.

\section{Conclusiones}

Los aceites esenciales de B. graveolens y L. meyenii presentan poco o nulo efecto sobre las larvas de las tres especies de crisópidos estudiados; mientras que, el aceite esencial de $M$. communis, presenta efecto larvicida en los controladores biológicos utilizados. $B$. graveolens y L. meyenii, serían factores importantes para considerar en el MIP, ya que podría prevenirse su afectación sobre los controladores biológicos. Además, se debe ampliar los ensayos en laboratorio y en campo para verificar que no exista interferencia entre el uso de los aceites esenciales sobre el desarrollo y la actividad de los neurópteros como controladores biológicos en el MIP. 


\section{Referencias}

Aldana, L., Salinas, D., Valdés, M., Gutiérrez \& Valladares, $M$. (2010). Evaluación bioinsecticida de extractos de Bursera copallifera (D.C.) Bullock y Bursera grandifolia (Schtdl.) Engl. en gusano cogollero Spodoptera frugiperda J.E. Smith (Lepidoptera: Noctuidae). Polibotánica, 29, 149-158.

Alegre, A., Bonifaz, E., Solange, S. \& Iannacone, J. (2017a). Sensibilidad de dos biocontroladores Chrysoperla externa y Chrysoperla carnea (Neuroptera: Chrysopidae) frente al extracto acuoso de Ruta graveolens (Rutacea). The Biologist (Lima), 15, 173-180.

Alegre, A., Iannacone, J. \& Carhuapoma, M. (2017b). Toxicidad del extracto acuoso, etanólico y hexánico de Annona muricata, Minthostachys mollis, Lupinus mutabilis, y Chenopodium quinoa sobre Tetranychus urticae y Chrysoperla externa. Chilean Journal of Agriculture and Animal Science, ex Agro-Ciencia, 33(3), 273-284.

Arias, J., Sila, G., Figueroa, I., Fischer, S., Robles, A., Rodríguez, J. \& Lagunes, A. 2017. Actividad insecticida, repelente y antialimentaria del polvo y aceite esencial de frutos de Schinus molle L. para el control de Sitophilus zeamais (Motschulsky). Chilean Journal of Agriculture and Animal Science, ex Agro-Ciencia, 33, 93-104.

Ayvaz, A., Albayrak, S. \& Karaborklu, S. 2008. Sensibilidad a la radiación gamma de los huevos, larvas y pupas de la polilla de la comida india Plodia interpunctella (Hübner) (Lepidoptera: Pyralidae). Ciencia de manejo de plagas, 64, 505-512.

Ayvaz, A., Sagdic, O., Karaborklu, S. \& Ozturk, I. 2010. Insecticidal activity of the essential oils from different plants against three stored-product insects. Journal of Insect Science, 10, 21-30.

Bakkali, F., Averbeck, S., Averbeck, D. \& Idaomar, M. (2008). Biological effects of essential oils- A Review. Food and Chemical Toxicology, 46, 446-475.

Barrueta, L.O., Martín, V.C., Castellanos, G.L. \& Jiménez, C.R. (2017). Extracto acuoso de Euphorbia lactea Haw como alternativa local para el control de Plutella xylostella L.. Revista Centro Agrícola, 44, 49-55

Berka-Zougali, B., Ferhat, M., Hassani, A., Chemat, F. \& K. Allaf. (2012). Comparative study of essential oils extracted from Algerian Myrtus communis L. leaves using microwaves and hydrodistillation. International Journal of Molecular Sciences, 13(4), 4673-4695.

Bussmann, R. W. \& Sharon, D. (2015). Plantas Medicinales de los Andes y la Amazonia, la flora mágica y medicinal del norte del Perú. Centro William L. Brown - Jardín Botánico de Missouri, Trujillo, Perú.

Campos, E., De Oliveira, J., Pascoli, M., De Lima, R. \& Fraceto, L. (2016). 
Neem oil and crop protection: from now to the future. Frontiers in Plant Science, 7, e1494.

Castillo, R. P. 2004. Estudio químico y de actividad antioxidante en Lepechinia meyenii.

Tesis Magister en Química. Pontifica Universidad Católica del Perú, Lima, Perú.

Celaya, M. H., Anaya, I.J., Barrera, S.M., Barrales, H.S., Nieblas, L.M. \& Osuna, C.R. (2019). Extractos hidro-etanólicos de plantas comestibles como alternativa para controlar bacterias patógenas, parásitos e insectos en la industria pecuaria. Biotecnia Revista de Ciencias Biológicas y de la Salud, 21, 47-54

Choi, W., Lee, E., Choi, B., Park, H. \& Ahn, Y. (2003). Toxicity of plant essential oils to Trialeurodes vaporariorum (Homoptera: Aleyrodidae). Journal of Economic Entomology 96, 1479-1484.

Clemente, S., Mareggiani, G., Broussalis, A. \& Ferraro, G. (2007). Actividad insecticida de 1,8-cineol sobre mosca delos frutos, Ceratitis capitata Wied. (Diptera: Tephritidae). Dominguezia, 23, 29-34.

Cruz G. S., Wanderley-Teixeira, V., Oliveira, J.V., Correia, A.A., Breda, M.O., Alves, T.J., Cunha, F.M., Teixeira, A.A., Dutra, K.A. \& Navarro, D.M. (2014). Bioactivity of Piper hispidinervum (Piperales: Piperaceae) and Syzygium aromaticum (Myrtales: Myrtaceae) oils, with or without formulated Bta on the biology and immunology of Spodoptera frugiperda (Lepidoptera: Noctuidae). Journal of Economic Entomology, 107, 144-153.

Fusé, C. B., Villaverde, M.L., Padín, S.B., De Giusto, M. \& Juaréz, M.P. (2013). Evaluación de la actividad insecticida de tierras de diatomeas de yacimientos argentinos. RIA. Revista de Investigaciones Agropecuarias, 39, 207-213.

García, V., Soto, A. \& Bacca, T. (2014). Efecto insecticida de productos alternativos en Trialeurodes vaporariorum (Hemiptera: Aleyrodidae). Revista Colombiana de Entomología, 40,143-147.

Gardeli, C., Vassiliki, P., Athanasios, M., Kibouris, T. \& Komatis, M. (2008). Essential oil composition of Pistacia lentiscus L. and Myrtus communis L.: Evaluation of antioxidant capacity of methanolic extracts. Food Chemistry, 107, 1120-1130

Gonzáles, O. \& Reguillón, C. (2002). A new species of Chrysoperla (Neuroptera: Chrysopidae) from Argentina. Revista de la Sociedad Entomologica Argentina, 61, 47-50.

González, R., Silva, G., Urbina, A. \& Gerding, M. (2016). Aceite esencial de Eucalyptus globulus Labil y Eucalyptus nitens H. Deane \& Maiden (Myrtaceae) para el control de Sitophilus zeamais Motschulsky. Chilean Journal of Agricultural \& Animal Sciences (ex Agro-Ciencia), 32, 204-216. 
Guzmán, J. (2005). Propagación in vitro de especies pertenecientes a las familias Rosaceae (Hesperomeles goudotiana y Rubus glaucus), Ericaceae (Macleania rupestris, Vacinium meridionale) y Cactaceae (Opuntia ficus indica). Informe técnico. Bogotá: Jardín Botánico José Celestino Mutis.

Iannacone, J. \& Lamas, G. (2002). Efecto de dos extractos botánicos y un insecticida convencional sobre el depredador Chrysoperla externa. Manejo Integrado de Plagas y Agroecología 65, 92-101.

Iannacone, J. \& Lamas, G. (2003). Efectos toxicológicos de extractos de molle (Schinus molle) y Lantana (Lantana camara) sobre Chrysoperla externa (Neuroptera: Chrysopidae), Trichogramma pintoi (Hymenoptera: Trichogram-matidae) y Copidosoma koehleri (Hymenoptera: Encyrtidae) en el Perú. Agricultura Técnica, 63, 347-360.

Iannacone, J. \& Alvarińo, L. (2005). Selectividad del insecticida cartap empleando bioensayos con organismos no destinatarios. Ecología Aplicada, 4, 91-104.

Iannacone, J. \& Alvariño, L. (2010). Toxicidad de Schinus molle L. (Anacardiaceae) a cuatro controladores biológicos de plagas agrícolas en el Perú. Acta Zoológica Mexicana, 26, 603-615.

Iannacone, J., Alvarińo, L., La Torre, M.I., Guabloche, A., Ventura, K., Chero, J., Cruces, C., Romero, S., Tuesta, E., Saez, G., MacDonald, D., Tueros, G., Argota, G., Fimia,
R. \& Carhuapoma, M. (2015). Toxicidad aguda y crónica de Tagetes elliptica (Asteraceae) y dimetoato sobre depredadores y parasitoides de plagas de importancia agrícola en Perú. The Biologist (Lima), 13, 329-347.

Khani, A. \& Basavand, F. (2012). Chemical composition and insecticidal activity of myrtle (Myrtus communis L.) essential oil against two stored-product pests. Journal of Medicinal Plants and By-products, 2, 83-89.

Kostyukovsky, M., Rafaeli, A., Gileadi, C., Demchenko, N. \& Shaaya, E. (2002). Activation of octopaminergic receptors by essential oil constituents isolated from aromatic plants: possible mode of action against insect pests. Pest Management Science 58, 1101-1106.

Lee, S., Peterson, C. \& Coats, J. (2002). Fumigation toxicity of monoterpenoids to sever al stored product insects. Journal of Stored Products Research, 39, 77-85.

Leyva, M., Martínez, J. \& Stashenko, E. (2007). Composición química del aceite esencial de hojas y tallos de Bursera graveolens (Burseraceae) de Colombia. Scientia et Technica, 33, 201-202.

Leyva, M., Tacoronte, J., Marqueti, M., Scull, R., Tiomno, O., Mesa, A. \& Montada, D. (2009). Utilización de aceites esenciales de pinaceas endémicas como una alternancia en el control del Aedes aegypti. Revista Cubana de Medicina Tropical, 61, 239-243. 
Martínez, Y., Castellanos, L. \& Ortega, I. (2014). Efecto insecticida de extractos de plantas para el control de áfido de la habichuela en la Empresa Azucarera Elpidio Gómez. Agroecosistemas, 2, 208-214.

Martins, A. \& Valle, D. (2012). The Pyrethroid Knockdown Resistance. En: Soloneski, S. \& Larramendy, M. (eds.) Insecticides-Basic and Other Applications. InTech Publication, pp. 17-38.

Medina, P., Budia, F., Del Estal, P. \& Viñuela, E. (2004). Influence of azadirachtin, a botanical insecticide, on Chrysoperla carnea (Stephens) reproduction: toxicity and ultrastructural approach. Journal of Economic Entomology, 97, 43-50.

Motazedian, N., Ravan, S. \& Bandani, A. (2012). Toxicity and repellency effects of three essential oils against Tetranychus urticae Koch (Acari: Tetranychidae). Journal of Agricultural Science and Technology, 14, 275-284.

Moura, P., Carvalho, A. \& Botton, M. (2012). Residual effect of pesticides used in integrated apple production on Chrysoperla externa (Hagen) (Neuroptera: Chrysopidae) larvae. Chilean Journal of Agricultural Research, 72, 217.

Muñoz, J., Staschenko, E. \& Ocampo, O. 2014. Actividad insecticida de aceites esenciales de plantas nativas contra Aedes aegypti (Diptera: Culicidae). Revista Colombiana de Entomología, 40, 198-202.
Núñez, Z.E. (1988). Ciclo biológico y crianza de Chrysoperla externa y Ceraeochrysa cincta (Neuroptera: Chrysopidae). Revista Peruana de Entomología, 31, 76-82.

Papachristos, D. \& Stamopoulos, D. (2004). Fumigant toxicity of three essential oils on the eggs of Acanthoscelides obtectus (Say) (Coleoptera: Bruchidae). Journal of Stored Products Research, 40, 517-525.

Parolín, P., Bresch, C. \& Poncet, C. (2015). Biocontrol plants and functional diversity in biological control of the red spider mite Tetranychus urticae: A review. International Journal of Agricultural Policy and Research, 3, 198-212.

Quispe, S.M. \& Tenorio, R.E. (2018). Determinación fitoquímica y actividad antimicrobiana del aceite esencial de los órganos aéreos de Lepechinia meyenii (Walp.) Epling "pacha salvia". Tesis para título profesional de químico farmacéutico. Universidad Wiener, Lima, Perú.

Ramírez, D.M., López, A.I., González, H.A. \& Badii, Z.M. (2007). Rasgos biológicos y poblacionales del depredador Cereaeochrysa sp. nr. cincta (México) (Neuroptera: Chrysopidae). Acta Zoológica Mexicana, 23, 79-95.

Ramírez, J., Gómez, M., Cotes, J. \& Nústez, C. (2010). Efecto insecticida de los aceites esenciales de algunas lamiáceas sobre Tecia solanivora Povolny en condiciones 
de laboratorio. Agronomía Colombiana, 28, 255-263.

Rasooli, I., Moosavi, M., Rezaee, M. \& Jaimand, K. (2002). Susceptibility of microorganisms to Myrtus communis L. essential oil and its chemical composition. Journal of Agricultural Science and Technology, 4, 127-133.

Rossi, C., Arias, G. \& Lozano, N. (2002). Evaluación antimicrobiana y fitoquímica de Lepechinia meyenii (Walp.) "Salvia". Instituto de Investigación en Ciencias Farmacéuticas y Recursos Naturales. Farmacia, Lima. Ciencia e investigación 5, Recuperado en http://www.sisbib.unmsm.edu.pe/ BvRevistas/ciencia/vol_5N1_2002/ evaluaci\%C3\% B3n_antimicro biana. htm (Consultado 20 mayo 2019)

Ruiz, C., Díaz, C. \& R. Rojas, R. (2015). Composición química de aceites esenciales de 10 plantas aromáticas peruanas. Revista de la Sociedad Química del Perú, 81(2), 81-94.

Sariol, S.D., Aponte, R.D. \& Hernández, M.L. (2019). Análisis fitoquímico $y$ actividad insecticida in vitro de extracto acuoso de Agdestis clematidea en el manejo de Myzus Persicae. Roca, 15, 28-38.

Sarwar, M. (2015). The killer chemicals for control of agriculture insect pests: the botanical insecticides. International Journal of Chemical and Biomolecular Science, 1, 123-128.

Satrani, B., Farah, A. \& Talbi, M. (2006). Fractionnal distillation effect on the chemical composition and antimicrobial activity of Moroccan Myrtle (Myrtus communis L.). Acta Botanica Gallica, 153, 235-242.

Shakarami, J., Kamali, K. \& Moharramipour, S. (2005). Fumigant toxicity and repellency effect of essential oil of Salvia bracteata on four species of warehouse pests. Journal of the Entomological Research Society, Iran, 24, 35-50.

Silva, A., Andrade, G., Freire, C. \& Bastos, D.. (2012). Effects of pesticides on eggs of Chrysoperla externa (Neuroptera: Chrysopidae) and consequences on subsequent development. Revista Colombiana de Entomología, 38, 58-63.

Souza, A. P., Marqués, M.R., Mahmoud, T.S., Caputo, B.A., Canhete, G.M., Leite, C.B. \& De Lima, D.P. (2008). Bioprospecting insecticidal compounds from plants native to Mato Grosso do Sul, Brazil. Acta Botanica, 22, 1136-1140.

Tarqui, J. (2007). Efecto de tres bioplaguicidas para el control del pulgón (Aphis sp.) en el cultivo de lechuga en ambientes protegidos en la ciudad de El Alto. Trabajo de Diploma en opinión al título de Ingeniero agrónomo. Universidad Mayor de San Andrés, Bolivia.

Tayoub, G., Abu, A. \& Ghanem, I. (2012). Fumigant activity of leaf essential oil from Myrtus communis L. against the Khapra beetle. International Journal of Medicinal and Aromatic Plants, 2, 207-213. 
Tuberoso, C., Barra, A., Angioni, A., Sarritzu, E. \& Pirisi, F. (2006). Chemical composition of volatiles in Sardinian myrtle $(M$. communis L.) alcoholic extracts and essential oils. Journal of Agricultural and Food Chemistry, 54, 1420-1426.

Unión Internacional para la Conservación de la Naturaleza (UICN). (2019). UICN - Especies para Restauración. Disponible en http://www. especiesrestauracion-uicn.org/data especie.php?sp_name $=$ Bursera $\% 20$ graveolens. (Consultado el 16 mayo 2019)

Valdiviezo, C.E. (2019). Actividad biocida del aceite esencial y extractos vegetales de Eucalyptus globulus L. y Artemisia absinthium L. obtenidos de la región $\mathrm{La}$ Libertad sobre adultos de Aedes aegypti. Tesis de Maestría, Universidad Nacional de Trujillo, Trujillo, Perú.

Weber, I.C. (1993). Methodsfor measuring the acute toxicity of effluents and receiving waters to freshwater and marine organisms. EPA/600/490/027F. 310Zayzafoon, G., Odeh, A. \& Allaf, W. (2011). The use of photochemiluminescence assay for the measurement of integral antioxidant capacity in Syrian Myrtus comminus L. leaves. Herba Polonica, 57, 5-19. 
